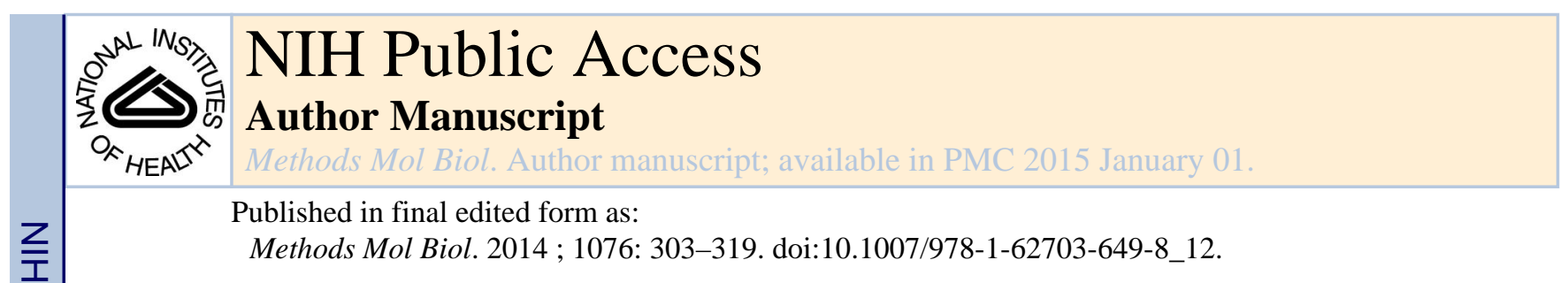

\title{
Upconversion Spectrophotofluorometry
}

\author{
Arianna Biesso, Jianhua Xu, and Jay R. Knutson
}

\section{Abstract}

As the other chapters attest, sensitivity of fluorescent molecules to their local environment has created powerful tools in the study of molecular biology, particularly in the study of protein, DNA, and lipid dynamics. Surprisingly, even events faster than the nanosecond lifetimes of fluorophores are important in protein function, and in particular, events lasting just a few ps reflect on water motion and the coupled dynamics of proteins. These ultrafast phenomena can best be studied by using the same laser that excites fluorescence to also "strobe" the emission, providing sub-picosecond time slices of the action. We explain the strobing "upconversion" technique and some limits on its execution.

\section{Keywords}

Time-resolved fluorescence; Upconversion; Tryptophan; Protein dynamics and photodamage

\section{Introduction}

\subsection{A Brief History of Upconversion Spectroscopy}

The newly discovered red ruby laser was focused into quartz in 1961 by Franken et al. [1], and the output beam was passed through a prism spectrograph, allowing them to observe a much weaker $347 \mathrm{~nm}$ (doubled frequency) spot. While frequency mixing of many types was known in microwave circuits, RF cavities, and (of course) low-frequency electronics (esp. household radio heterodyne circuit stages), optical mixing had not previously been seen.

The other nonlinear optical phenomena followed, including higher harmonic generation and generation of both optical difference frequency (e.g., for novel monochromatic IR beams) and sum frequency generation. The latter, conceptually identical to second harmonic generation, gained favor as a method to convert weak infrared signals into visible signals amenable to detection with more conventional cameras and detectors. The redder signal was said to have been frequency "upconverted" into the visible.

To accomplish upconversion, a stronger (and usually redder) optical field was needed to mix with the weak signal. Just as in electronics, mixing demands the voltages be high enough to elicit a distorted (nonlinear) response of the mixer material to the sinusoidal waves.

Otherwise, the beams simply superimpose in the crystal, and the electrons simply track the superposition of electric fields. The strong electric fields of short laser pulses were just what was needed to reveal second-order crystal nonlinearity and mixing. Of course, to create mixing, the electric fields had to superimpose not only in space, but also in time. This created the opportunity to auto or cross-correlate laser pulses, since the mixed signal output envelope is a temporal convolution of the input pulses. 
Autocorrelation and cross-correlation of pulses set the stage for not only transforming wavelengths, but also quantifying ultrafast kinetics. The photocathodes all found at the heart of photomultipliers, vacuum diodes, and streak cameras invariably produce a spread of photoelectron ejection energies in response to a laser pulse, meaning those electrons take slightly different paths (and times) to their eventual detection. As a result, phenomena faster than several ps were intractable for even the fastest photodetectors. Optical mixing was the best solution.

In chemistry, the first published use of upconversion to trace a luminescent process was by Halliday and Topp [2]. The method spread rapidly in photophysics, as briefly reviewed by Barbara and colleagues [3].

Ultrafast spectroscopy (particularly time-resolved fluorescence) has been a fruitful area of biological research. While it is, at first, counterintuitive to think that nanosecond (or worse, picosecond and faster) events bear relevance to enzymatic and structural changes taking a billion times longer, the facts have been clear: changes in the fluorescent signals on or in proteins, membranes, and DNA give intimate details about the size, flexibility, fluidity, and interrelationships (including proximity and motion) of these various macromolecules. Several other chapters in this book will provide examples of this windfall.

Among the various biophysical targets of interest, upconversion spectroscopy quickly became most popular in a field that, unsurprisingly, is hungriest for time-resolved data tracing the flow of photonic energy: Photosynthesis. The study of photosynthetic pigments and their photochemical and functional physical arrangements includes relevant kinetics on everything from femtosecond through minute timescales [4-6].

\subsection{Use of Upconversion to Study Proteins}

Tryptophan is both the blessing and curse of protein dynamics spectroscopy. Present in most proteins, yet sparse enough that most have only a few $\operatorname{Trp}(\mathrm{W})$ in their sequence, it represents a local reporter that is intricately linked to its surroundings as it performs its native structural and/or electronic duties [7]. Trp has been studied for years with timeresolved spectroscopy on the nanosecond scale, and the best software and genetic manipulations have been applied to the task of isolating the signals belonging to single Trps within proteins $[8,9]$. Such signals tell us about local mobility and folding, overall tumbling of the protein, proximity to FRET acceptors such as NADH [10] or heme [11], and the availability of the Trp ring to solvent quenchers [12].

Single Trp in proteins often provides multiexponential decay behavior, a fact that had led to a long-standing controversy about why the protein environment is not homogeneous on that timescale. Two camps evolved, one (observing comparable features of indole in glycerol) and seeing Trp in proteins also tended to have longer decays at the red side of the spectrum, invoked ns solvent relaxation [13,14]. A second camp invoked Occam's razor to point out the decay surface was compatible with simple heterogeneity-perhaps by mechanisms as simple as side-chain rotamers (or more broadly, conformers) $[15,16]$. The nanosecond fluorometry "heterogeneity vs. relaxation" debate went on throughout the last three decades, and the combination of studies on crystals (where orientation modified preexponential 
weights, a result incompatible with relaxation) [17] and studies on constrained Trp derivatives [18] pushed toward a consensus that on the nanosecond scale, Trp decay in proteins was often dominated by heterogeneity.

This was not to say relaxation was absent; instead, it was suggested that it was mostly complete within $\sim 100 \mathrm{ps}$ (the lower time resolution typical of nanosecond instruments). Most telling was the absence of a negative preexponential termon the red side of the Trp emission, a signal known to flag relaxation (or any other excited state reaction) [19]. Advocates of relaxation models, while agreeing this rising term should be present, rightly argued that the negative preexponential could be quite small and therefore easily lost in either noise or the contributions of short-lived contaminants (perhaps photoproducts) [20].

A progression of nanosecond lifetimes associated with a progression to the red in spectral position (redder "DAS" correlating with longer lifetime) was also suggested as reflective of relaxation, and the paucity of proteins breaking that pattern was noted. Only recently, the intrinsic nature of the nanoheterogeneous quenching mechanism has been found to favor this correlation [21].

Early picosecond studies of Trp, meanwhile, were carried out laser-based chemical physics community, and they were generally remote from the nanosecond Trp controversy. Treating Trp like other dyes (e.g., coumarins) in solvents, they obtained time-dependent fluorescence Stokes shifts (TDFSS) (or fluorescence dynamic Stokes shift, FDSS) and deduced correlation functions, concerned primarily with the spectral movement rather than deactivation [22, 23]. Exceptions to this focus were studies of Trp in heme-containing proteins, where efficient FRET was anticipated from Trp to Soret bands, leading to sub -30 ps overall lifetimes [24, 25]. The early behavior of Trp was also probed for emission anisotropy [26], where, unfortunately, some Raman contamination to curves (apparently) made Trp appear to be a complex, vibrational-cooling dominated system (likely too complex to be useful in ps motion studies). In the late 1990s, however, Shen et al. [27] showed that Trp not only produced strong $1.6 \mathrm{ps}$ solvent relaxation spectra in water, but also had simple anisotropy behavior; oscillators within Trp analogs were seen to perform internal conversion (reaching stable orientation) well within 100 fs. This opened the door to exploiting Trp without the complexity concerns previously mentioned.

A recurrent theme in protein dynamics is the protein-water interface. Data available from Xray to neutron diffraction point to an ordered water layer in direct contact with the protein. The dynamic properties of this ordered layer of water are not clear, and if they are different it poses a question: Are these water molecules simply slaved to the protein charge distribution and dynamics or does the ordered water also affect protein dynamics? The nuclear overhauser effect (NOE) allows the investigator to probe water relaxation and compare it to bulk water with sub-nanosecond time resolution; further, nuclear magnetic relaxation dispersion (NMRD) has shorter effective time resolution. FDSS, using fluorescence upconversion on Trp residues of Monellin, showed spectral downshifting that was slower compared to those observed for single indole molecules in solution. It was then proposed that such a slow (8-20× slower) shift was the signature of a layer of organized water (also called "biological water"). This new "slow" water on protein would have 
solvation dynamics in the range of 30 to hundreds of picoseconds, in contrast to the few picoseconds measured for bulk water. In protein dynamics, X-ray diffraction can provide initial structures that, along with MD simulation, can predict stepwise information on this layer of solvation water. Recent results have also been gleaned from ${ }^{17} \mathrm{O}$ spin relaxation, providing values for the dynamics of water at the interface that are consistently faster that those inferred using FDSS.

When the laboratory of laureate A. Zewail published relaxation profiles for Trp within the sweet protein Monellin, finding not only the $1.6 \mathrm{ps}$ "bulk water" relaxation term but also a redshift that required $10 \mathrm{~s}$ of ps, they (as mentioned above) attributed this slower process to exchanging water between bulk and bound sites with off-rates near 20 ps [28]. Importantly, they also made emission anisotropy measurements in a variety of protein states, confirming the viability of Trp as a probe of segmental motion [29, 30].

Noting that the raw TRES (Time Resolved Emission Spectra) of Monellin did not rise after a few ps, Xu et al. [45] showed that this 20 ps behavior of Monellin was actually dominated by an ultrafast quenching process (the blue side was simply being consumed more rapidly than red) rather than a conservative steady redshift like that seen in bulk water. In other words, no negative preexponential was found for the 20 ps term. This essentially caused the two model controversy from ns Trp studies to reemerge at $100 \times$ faster rates.

Other proteins $[31,32]$ clearly did show the negative amplitude feature. Was relaxation always present and was it simply "masked" in some proteins by superposed quenching? Was it unique to proteins with particular folds, partial unfolding, or denaturation? Monellin in denaturants was certainly a strong exhibitor of $\sim 50$ ps relaxation, but the presence of a viscous denaturant complicates any dynamics gleaned from that picture [28].

One approach to the "masking" question was mutagenesis—by removing likely quenchers in the neighborhood of Trp (and replacing them with benign side chains), a quencher-free relaxation was seen [33].

Meanwhile, the rapid quenching, naturally unseen in nanosecond instrumentation, was termed QSSQ (Quasi-Static Self Quenching) in accordance with Chen et al. and it was found to be effective even in simple dipeptides [34, 35]. More importantly, it was seen that there were not only $<100$ ps quenching rates, but also losses faster than the instrument response of $0.2 \mathrm{ps}$ (200 fs). The "initial brightness" (taken typically at $\sim 1 \mathrm{ps)}$ could be used to sense this, and the sum of these ultrafast terms reconciled yield defects in a variety of peptides [36]. An actual biological use for QSSQ was found in the eye lens proteins termed crystallins; apparently, animals have evolved to keep these proteins from oxidizing in ultraviolet light by creating very strongly quenched Trp environments [37]. Thus, the ultrafast QSSQ is a useful probe of proteins independent of the relaxation phenomenon it masks. 


\subsection{Molecular Dynamics (MD) as a Tool to Decode Protein Dynamics}

Molecular dynamics (MD) simulations have contributed in shaping today's awareness of proteins acting as dynamic complex systems, and they also provide a unique window on the behavior of protein and solvation water in the femto- to picosecond timescale.

Attempts to follow protein dynamics involving the use of covalently bound extrinsic fluorescence probes produced mixed results. In some cases it was difficult to deconvolute the protein internal dynamics from those of the dye [38]. The protein dynamics community pursued many different routes. Alongside fluorescence dynamic Stokes shift (FDSS), a number of different techniques were employed to probe protein dynamics, esp. NMR and nuclear overhauser effect (NOE) with MD simulations performed in parallel [39-42].

Decoding of the Trp fluorescence signal and its correlation to short- and long-range molecular dynamics in proteins is not an easy task. MD simulations proved to be essential in helping with the data interpretation; hybrid QM-MM simulation methods have indeed been successful in predicting fluorescence emission maxima and quantum yields for Trp residues in a variety of proteins $[43,44]$. Importantly, the study done on $\gamma \mathrm{S}$ and $\gamma \mathrm{D}$-crystallin revealed multiexponential decays in the ps time domain along with a very rapidly quenched population, and QM-MM simulations done on such residues clearly disclosed that nearby charges were the cause for the fast quenching: A Trp charge transfer (CT) state was produced with the amide backbone, stabilized by nearby water and charged residues; thus, the predicted Trp fluorescence lifetime was drastically reduced [37]. As mentioned above, nature might have selected such "enhanced" quenching mechanisms in crystallin to insure longevity in an eye lens proteins heavily exposed to UV light.

The most recent work in this area by our group concludes that relaxation is ubiquitous but can be masked by the equally valuable QSSQ phenomenon. We find relaxation in GB1 is remarkably refractory to $\mathrm{pH}$-induced changes in protein structure that have profound effects on ns emission [45]. Further, substitution of Trp analogs via auxotrophic biosynthesis can eliminate the QSSQ term. For example, relaxation within the aforementioned Monellin protein (dominated by 20 ps quenching) is "unmasked" when 5FTrp replaces Trp [46].

Upconversion of Trp then gives us access to two different valuable indicators of protein dynamics: relaxation, with wider extent, and QSSQ, with precise dependence on Trp nearest neighbors.

\section{Materials}

\subsection{Upconversion Spectrophotofluorometer and Experimental Instructions}

Fluorescence upconversion is a derivative of the nonlinear pump probe technique, and a spectrophotofluorometer was developed especially for studying tryptophan fluorescence dynamics in proteins, which is shown in Fig. 1. A $\sim 400 \mathrm{~mW}$ pulse train with a typical pulse duration of $120 \mathrm{fs}$ and a repetition rate of $82 \mathrm{MHz}$ was generated from a mode-locked Ti: sapphire laser (Tsunami, Spectra-Physics) pumped by a diode-pumped solid state (DPSS) laser with the power of $5 \mathrm{~W}$ (Millennia, Spectra-Physics). It seeds a Ti: sapphire regenerative amplifier (Spitfire, Spectra Physics) pumped by a diode-pumped nanosecond 
green laser with average power of $15 \mathrm{~W}$ at $5 \mathrm{kHz}$ (Empower, Spectra-Physics). The output amplified pulses at $885 \mathrm{~nm}$ typically had a pulse energy of $0.2 \mathrm{~mJ}$ and an autocorrelation pulse width of $350 \mathrm{fs}$ (see Fig. 2) at a repetition rate of $5 \mathrm{kHz}$ (i.e., $\sim 1 \mathrm{~W}$ output power). Ultraviolet excitation pulses at $295 \mathrm{~nm}$ with an average power up to $10 \mathrm{~mW}$ were obtained from nonlinear harmonic generation (a $1 \mathrm{~mm} \mathrm{BBO}$ crystal and $0.5 \mathrm{~mm} \mathrm{BBO}$ crystal for doubling and tripling, respectively). This UV beam was separated from the infrared beam (fundamental) and visible (doubled) by two dichroic mirrors and by spatial filtering (using a pinhole). Power should be carefully attenuated to below $1 \mathrm{~mW}$ before excitation of the sample to avoid photodegradation, hole burning, and other undesirable effects. A zero-order half wavelength plate (HWP) was put on a computer-controlled servo stage and used to change the polarization of the UV beam. A circular array of thin cells (T-20, NSG Precision Cells) with a path length of $1 \mathrm{~mm}$ in a delrin stacked slotted disk was used to hold samples, and the total volume was about $2 \mathrm{ml}$. The disk was spun continuously (up to $5 \mathrm{~m} / \mathrm{s}$ circumferential motion) to make each UV pulse excite a slightly different sample spot. The remaining infrared fundamental pulse was reflected from a broadband hollow retroreflector on a computer-controlled precision stage and used as a gate pulse for the upconversion process. The fluorescence emission was collected by parabolic mirrors, passed through a long pass filter $(\mathrm{F})$, and focused into a $0.5 \mathrm{~mm}$ thick BBO mixing crystal. The upconversion signal was produced via type I sum frequency generation with the gate pulse in the crystal. Here the combination of a thin BBO crystal and mirrors is selected for reducing the limitations of time resolution imposed by group velocity mismatch and dispersion. The time evolution of fluorescence at any given wavelength can be obtained by using a computer to set the $\mathrm{BBO}$ crystal angle, monochromator wavelength (at $\left.\omega_{\mathrm{F}}+\omega_{\mathrm{p}}\right)$, and scan delay stage. The delay stage is comprised of a hollow cube corner retroreflector (Newport UBBR1-5I) mounted on a cross roller slide (Parker) linear stage driven by compumotor motor, combined with appropriate NIR relay mirrors.

To reject the strong background signals (infrared laser and its second harmonic generation, remnant UV, and unconverted fluorescence) accompanying the upconverted signal, a nonconfiguration was arranged between infrared probe and fluorescence. The use of a monochromator helps in reducing these unwanted signals at the PMT, also improving the spectral bandwidth. A double monochromator may be necessary if this problem is severe. Polarizations of gated fluorescence were determined by the orientation of nonlinear crystals, so no extra linear polarizer was needed (for anisotropy calculation, the grating factor $\mathrm{G} \sim 1$ ). By angle tuning the mixing crystal, the upconverted fluorescence signal, with a wavelength in the range $230-280 \mathrm{~nm}$, always polarized in the same direction, was directed into a monochromator (Triax 320, Jobin Yvon Inc. with a bandwidth of $0.5 \mathrm{~nm}$ ) and a solar blind photomultiplier tube (R2078, Hamamatsu, dark rate <1 cps). Amplified SBPMT signals were discriminated and then recorded by a gated single photon counter (994, EG\&G Ortec). Photon arrival events were held to less than $5 \%$ of the repetition rate to minimize "pileup" (which might flatten transient). One should mention that there are two main sources of noise for the detection. If the signal is sufficiently greater than the PMT dark current, the power fluctuations of the infrared laser beam may be the primary noise source. If the signal is very low, the shot noise from the dark current of the PMT will be the limiting factor, and in this case, cooling the PMT is advisable. 
A precise determination of the bandwidth and zero time delay is essential in all ultrafast experiments. The best way of determining the zero in upconversion system is to get a cross correlation trace between the scattered laser light from the sample and a delayed laser beam in the crystal. Such a trace provides not only an accurate zero but also an accurate measurement of the system response time. In our system, the "lamp" (AKA "apparatus" or "instrument response") function was determined by measuring the cross-correlation trace either between the UV and infrared pulses or UV-generated spontaneous Raman scattering in water and the infrared. In both ways, the lamp function was found to be around $400 \mathrm{fs}$ (FWHM) shown in Fig. 2, with a timing jitter of less than 30 fs. The use of unamplified pulses and a more collinear geometry can potentially reduce this FWHM to $<150 \mathrm{fs}$, with at much lower selectivity.

When collecting on longer timescales, we often found focal "detuning" to $~ 1$ ps FWHM optimized other geometric parameters. Instrument calibration was further verified with indoles and, e.g., the linear fluorophores like $p$-terphenyl, which yielded $r_{0}=0.40 \pm 0.01$ and a single rotational correlation time $\phi$ of $41 \mathrm{ps}$ in cyclohexane. A schematic of the upconversion setup is shown below.

\section{Methods}

After tripling our $885 \mathrm{~nm}$ pulses, we can obtain an average energy of $5 \mu \mathrm{J}$ at $295 \mathrm{~nm}$, but we only use $0.2 \mu \mathrm{J}$.

The upconversion process itself, while linearly dependent on both IR power and fluorescence, is quite inefficient $\left(\sim 10^{-4}\right)$ and therefore, lasers with higher repetition rates and IR "gate" pulse powers are certainly to be desired.

\subsection{Upconversion Sample Preparation and Data Collection}

1. Degas the buffer of choice beforehand. Although this step is not strictly necessary, it will improve the sample photostability upon UV exposure.

2. Dissolve the biomolecule of interest in the buffer to a concentration of about 0.5$0.8 \mathrm{mM}$ depending on the quantum yield (QY) of the Trp under analysis. Concentrations as low as $150 \mu \mathrm{M}$ have been used for single Trp containing molecules with QYs around 0.3.

3. Absorption spectra must be taken before and after upconversion measurement to check for photodamage; in particular, one looks for loss of native Trp absorbance using the band at $280 \mathrm{~nm}$.

4. The solution is loaded on the sample holder (see Fig. 3) minimizing the amount of air bubbles. One of the six embedded cuvettes is usually filled with buffer only for stopping to selectively collect the system response function (AKA lamp function) from spontaneous Raman in situ.

5. When starting "cold," the first step to get an upconversion signal is to directly mix (in the BBO crystal) the $295 \mathrm{~nm}$ excitation with the $885 \mathrm{~nm}$ output, as these are powerful beams $(\sim 100 \times$ more than the divergent fluorescence emission from the 
sample) and it will be easier to use them to test the alignment of beams inside the crystal as well as benchmark the crystal angle.

6. Set the monochromator to the upconversion (quadrupling, in this case) wavelength of $221 \mathrm{~nm}$, attenuate the UV tenfold, and open the monochromator slits and look for the signal by scanning delays to match pathlenghts. To make sure that the source of your signal is in fact from 295 to 221 upconversion, move the delay line a few ps, and the impulse signal should disappear.

7. Once the signal is optimized, close the entrance slit so that you still have $\sim 80 \%$ signal and repeat the alignment optimization one more time.

8. Put the fluorescence standard sample in place, add about 10 ps delay, spin the disk array, and rotate the BBO to again look for an upconversion signal; here it is easier to start with the sample emission maximum $\sim 350 \mathrm{~nm}$ creating a signal near $251 \mathrm{~nm}$. Also remember when retracing the "lamp" temporal profile with disk stopped that the zero delay line will have to be moved; now that the $295 \mathrm{~nm}$ beam is going through the sample, it will be delayed a few picoseconds just by the difference in transit time between air and the silica-water sandwich now present. The same will be true whether observing 295 or the spontaneous Raman scatter "lamp" at 328 $\mathrm{nm}$. This makes the latter very useful.

9. After each run, to ascertain the system has time-invariant sensitivity, place a known long-lived monoexponential standard material (e.g., melatonin in $\mathrm{H}_{2} \mathrm{O}$ ) in the disk and collect repeated time traces until ca. 1000 photon counts are present at each interval (of several tens of points), yielding overall slope accuracy better than $1 \%$. Over short ( $<40 \mathrm{ps}$ ) intervals even ns decays appear level. If unexpected declines are seen, it is likely due to walk of the beam in the cube-corner delay stage, and one must realign using fine pinholes to detect any misalignment during stage movement.

10. Each trace should either be collected randomly in delay time or with averaging of both increasing and decreasing time traces, to reduce the influence of any bleaching or long-term laser intensity drift. Alternatively, one may recollect a few selected delay points to ascertain no drift/bleach reductions in intensity have occurred.

11. Since the traces provide photon counts vs. time, ordinary fitting routines for TCSPC data may be employed for fitting, or global analysis (preferred) can be applied to the entire data surface (several traces vs. wavelength).

12. The delay line requires calibration, which could be done by placing a slab of fused silica of known thickness $(d)$ and index of refraction $(n)$ on the path of the IR beam being careful not to disturb its positioning. The slab will create a delay $(D)$ equal to $d(n-1)$. Find the new zero delay. The ratio between $D$ and the number of steps needed to find the new zero will provide the delay imposed by each step

13. The number of counts per second obtained out of the sample should be much less than $10 \%$ of the repetition rate of the system. By doing so we reduce the probability of 2 photons reaching the detector simultaneously, a phenomenon called 
"pileup". This produces a measured count rate (see Graphs 1 and 2) significantly lower than the true count rate, according to Poisson statistics. The deviation from the true count rate is more severe in a system with lower repetition rate; this is one reason we upgraded our system to $5 \mathrm{kHz}$.

An example of a typical upconversion transient is shown in Fig. 4 after averaging five datasets; the number of laser flashes used exceeds the peak count by over $20 \times$.

\section{Notes}

\subsection{Tryptophan Photodamage}

As reviewed above Trp is a widely studied fluorescence probe, scientifically relevant for being the brightest fluorophore among amino acids, and largely used as a natural probe to report on a growing number of biological systems [47-50].

Photooxidation is the most common damage mechanism both in Trp and Tyr when irradiated with UV photons in an aqueous environment [51]. The oxidation mechanism is initiated by the formation of reactive oxygen species (ROS) along with hydroxyl radicals and singlet oxygen which ultimately will modify these residues into a variety of photoproducts [52]. There is an ongoing effort in understanding the photodegradation mechanism of Trp in aqueous solution, partly driven by the role played by photodegradation in the progression of a variety of diseases like yellowing of the human lens [53].

Fluorescence upconversion is a powerful technique that allows reporting on changes in the Trp microenvironment in the picosecond timescale, and as described in this chapter, the setup involves using short laser pulses (few hundred femtoseconds) in the UV range (280$295 \mathrm{~nm}$ ) in order to excite Trp to its first excited state (1La). In order to generate a detectable upconversion signal, less than $1 \mathrm{~mW}$ energy is sent into the sample. The energy is packed, however, in a few very short pulses, resulting in relatively high peak power being applied to the sample. In comparison to TCSPC measurements where the average energy is about 1 order of magnitude lower, for an upconversion type of measurement, the peak power applied to the sample is on average 4 orders of magnitude higher. These figures are a reminder that upconversion data are also more at risk for photodamage than other "linear" spectroscopies; as a result, more precautions need to be taken in preparing the sample like degassing the buffer, using oxygen scavengers, and avoiding reexcitation of the same volume.

We have found that some samples (particularly flexible peptides of 4-40 residues) are quite sensitive to the UV damage process during upconversion.

The generation of photoproducts in Trp-containing peptides was tested by taking multiple upconversion datasets over a $2 \mathrm{~h}$ period, after repeatedly exposing the sample to $295 \mathrm{~nm}$ excitation light. As shown in Fig. 5, remaining Trp lifetime at $375 \mathrm{~nm}$ is clearly shortened upon sample exposure to large doses of pulsed UV light. Electrospray ionization tandem mass spectroscopy (ESI-MS/MS) confirmed that oxidation took place almost exclusively at the Trp residue level. The take-home message is that photodamage can compromise fluorescence upconversion data resulting in an alteration of detected Trp fluorescence 
dynamics at the picosecond level, especially at the low energy side of the Trp emission spectrum.

A number of studies have been conducted on the mechanism and conditions that favor Trp photoproducts from UV light absorption, but the photophysics are not fully understood yet. Many of the proposed mechanisms point at photoproducts being mainly generated in the $S^{1}$ excited state over a nanosecond timescale. Some of these mechanisms invoke a proton transfer; others suggest that an electron transfer is involved.

Two of the best known products of Trp photodegradation are Kynurenine and NFormylkynurenine [54, 55]. Their absorption and emission spectra show a clear redshift compared to Trp; they also have intrinsically low fluorescence quantum yield, with NFormylkynurenine being a slightly better emitter between the two [56]. At the excitation wavelength of Trp, usually in the range of 285 to $295 \mathrm{~nm}$, a smaller amount of the photoproducts get excited. Whether they are directly observed in the milieu below/at $442 \mathrm{~nm}$ on the red edge, or whether they act as general fluorescence quenchers, a sub-nanosecond Trp lifetime definitely gets reduced (as shown in Fig. 5).

Recent investigations on Trp damage via UV irradiation uncovered that a large variety of photoproducts coexist with multiple oxidations taking place at the Trp residue (up to 4), providing a cascade of modifications [57]. Photodamage is an important artifact to carfully monitor in any laser study.

\section{References}

1. Franken PA, Weinreich G, Peters CW, et al. Generation of optical harmonics. Phys Rev Lett. 1961; 7:118-119.

2. Hallidy LA, Topp MR. Picosecond luminescence detection using type-2 phasematched frequencyconversion. Chem Phys Lett. 1977; 46:8-14.

3. Kahlow MA, Jarzeba W, Dubruil TP, et al. Ultrafast emission-spectroscopy in the ultraviolet by time-gated upconversion. Rev Sci Instrum. 1988; 59:1098-1109.

4. Bradforth SE, Jimenez R, Vanmourik F, et al. Excitation transfer in the core light-harvesting complex (LH-1) of rhodobacter- sphaeroides-an ultrafast fluorescence depolarization and annihilation study. J Phys Chem. 1995; 99:16179-16191.

5. Kennis JTM, Gobets B, van Stokkum IHM, et al. Light harvesting by chlorophylls and carotenoids in the photosystem I core complex of Synechococcus elongatus: a fluorescence upconversion study. J Phys Chem B. 2001; 105:4485-4494.

6. Macpherson AN, Gillbro T. Solvent dependence of the ultrafast S-2-S-1 internal conversion rate of beta-carotene. J Phys Chem A. 1998; 102:5049-5058.

7. Longworth, JW. Time-resolved fluorescence spectroscopy in biochemistry and biology. Plenum Press; New York: 1983.

8. Beechem JM, Brand L. Time-resolved fluorescence of proteins. Annu Rev Biochem. 1985; 54:4371. [PubMed: 3896124]

9. Christiaens B, Symoens S, Vanderheyden S, et al. Tryptophan fluorescence study of the interaction of penetratin peptides with model membranes. Eur J Biochem. 2002; 269:2918-2926. [PubMed: 12071955]

10. Schauerte JA, Schlyer BD, Steel DG, et al. Nanosecond time-resolved circularpolarization of fluorescence-study of NADH bound to horse liver alcohol-dehydrogenase. Proc Natl Acad Sci USA. 1995; 92:569-573. [PubMed: 7831331] 
11. Gryczynski Z, Lubkowski J, Bucci E. Heme-protein interactions in horse heart myoglobin at neutral $\mathrm{pH}$ and exposed to acid investigated by time-resolved fluorescence in the picosecond to nanosecond time range. J Biol Chem. 1995; 270:19232-19237. [PubMed: 7642594]

12. Eftink MR, Ghiron CA. Fluorescence quenching studies with proteins. Anal Biochem. 1981; 114:199-227. [PubMed: 7030122]

13. Lakowicz JR, Keating-Nakamoto S. Red-edge excitation of fluorescence and dynamic properties of protein and membranes. Biochemistry. 1984; 23:3013-3021. [PubMed: 6466628]

14. Alcala JR, Gratton E, Prendergast FG. Interpretation of fluorescence decays in proteins using continuous lifetime distribuition. Biophys J. 1987; 51:925-936. [PubMed: 3607213]

15. Szabo AG, Rayner DM. Fluorescence decay of tryptophan conformers in aqueous solution. J Am Chem Soc. 1980; 102:554-563.

16. Knutson JR, Beechem JM, Brand L. Simultaneous analysis of multiple fluorescence decay curvesa global approach. Chem Phys Lett. 1983; 102:501-507.

17. Dahms TES, Willis KJ, Szabo AG. Conformational heterogeneity of tryptophan in a protein crystal. J Am Chem Soc. 1995; 117:2321-2326.

18. Adams PD, Chen Y, Ma K, et al. Intramolecular quenching of tryptophan fluorescence by the peptide bond in cyclic hexapeptides. J Am Chem Soc. 2002; 124:9278-9286. [PubMed: 12149035]

19. Davenport L, Knutson JR, Brand L. Excited-state proton-transfer of equilenin and dihydroequilenin-interaction with bilayer vesicles. Biochemistry. 1986; 25:1186-1195. [PubMed: 3964666]

20. Toptygin D, Brand L. Spectrally- and time-resolved fluorescence emission of indole during solvent relaxation: a quantitative model. Chem Phys Lett. 2000; 322:496-502.

21. Pan CP, Muino PL, Barkley MD, et al. Correlation of tryptophan fluorescence spectral shifts and lifetimes arising directly from heterogeneous environment. J Phys Chem B. 2011; 115:3245-3253. [PubMed: 21370844]

22. Jimenez R, Fleming GR, Kumar PV, et al. Femtosecond solvation dynamics of water. Nature. 1994; 369:471-473.

23. Maroncelli M, Fleming GR. Picosecond solvation dynamics of Coumarin-153-the importance of molecular aspects of solvation. J Chem Phys. 1987; 86:6221-6239.

24. Janes SM, Holtom G, Ascenzi P, et al. Fluorescence and energy-transfer of tryptophans in aplysia mioglobin. Biophys J. 1987; 51:653-660. [PubMed: 3580491]

25. Gryczynski, Z.; Lubkowski, J.; Bucci, E. Intrinsic fluorescence of hemoglobins and myoglobins. In: Brand, L., editor. Fluorescence spectroscopy. Vol. 278. Elsevier Academic Press Inc; San Diego: 1997. p. 538-569.

26. Ruggiero AJ, Todd DC, Fleming GR. Subpicosecond fluorescence anisotropy studies of tryptophan in water. J Am Chem Soc. 1990; 112:1003-1014.

27. Shen XH, Knutson JR. Subpicosecond fluorescence spectra of tryptophan in water. J Phys Chem B. 2001; 105:6260-6265.

28. Peon J, Pal SK, Zewail AH. Hydration at the surface of the protein Monellin: dynamics with femtosecond resolution. Proc Natl Acad Sci USA. 2002; 99:10964-10969. [PubMed: 12177425]

29. Pal SK, Peon J, Zewail AH. Biological water at the protein surface: dynamical solvation probed directly with femtosecond resolution. Proc Natl Acad Sci USA. 2002; 99:1763-1768. [PubMed: 11842218]

30. Zhong DP, Pal SK, Zhang DQ, et al. Femtosecond dynamics of rubredoxin: tryptophan solvation and resonance energy transfer in the protein. Proc Natl Acad Sci USA. 2002; 99:13-18. [PubMed: 11752400]

31. Zhang LY, Wang LJ, Kao YT, et al. Mapping hydration dynamics around a protein surface. Proc Natl Acad Sci USA. 2007; 104:18461-18466. [PubMed: 18003912]

32. Qiu WH, Zhang LY, Okobiah O, et al. Ultrafast solvation dynamics of human serum albumin: correlations with conformational transitions and site-selected recognition. J Phys Chem B. 2006; 110:10540-10549. [PubMed: 16722765] 
33. Zhang LY, Kao YT, Qiu WH, et al. Femtosecond studies of tryptophan fluorescence dynamics in proteins: local solvation and electronic quenching. J Phys Chem B. 2006; 110:18097-18103. [PubMed: 16970418]

34. Chen RF, Knutson JR, Ziffer H, et al. Fluorescence of tryptophan dipeptides-correlation with the rotamer model. Biochemistry. 1991; 30:5184-5195. [PubMed: 2036384]

35. Chen Y, Liu B, Yu HT, et al. The peptide bond quenches indole fluorescence. J Am Chem Soc. 1996; 118:9271-9278.

36. Xu JH, Knutson JR. Quasi-static selfquenching of Trp-X and X-Trp dipeptides in water: ultrafast fluorescence decay. J Phys Chem B. 2009; 113:12084-12089. [PubMed: 19708715]

37. Xu JH, Chen JJ, Toptygin D, Tcherkasskaya O, et al. Femtosecond fluorescence spectra of tryptophan in human gamma-crystallin mutants: site-dependent ultrafast quenching. J Am Chem Soc. 2009; 131:16751-16757. [PubMed: 19919143]

38. Halder M, Mukherjee P, Bose S, et al. Solvation dynamics in protein environments: comparison of fluorescence upconversion measurements of coumarin 153 in monomeric hemeproteins with molecular dynamics simulations. J Chem Phys. 2007; 127:055101. [PubMed: 17688362]

39. Wang W, Donini O, Reyes CM, Kollman PA. Biomolecular simulations: recent developments in force fields, simulations of enzyme catalysis, protein-ligand, protein-protein, and protein-nucleic acid noncovalent interactions. Annu Rev Biophys Biomol Struct. 2001; 30:211-243. [PubMed: 11340059]

40. Halle B, Andersson T, Forsen S, et al. Protein hydration from water O-17 magneticrelaxation. J Am Chem Soc. 1981; 103:500-508.

41. Halle B, Wennerstrom H. Interpretation of magnetic-resonance data from water nuclei in heterogeneous systems. J Chem Phys. 1981; 75:1928-1943.

42. Roccatano D, Amadei A, Di Nola A, et al. A molecular dynamics study of the 41-56 beta-hairpin from B1 domain of protein G. Protein Sci. 1999; 8:2130-2143. [PubMed: 10548059]

43. Callis PR, Liu TQ. Quantitative prediction of fluorescence quantum yields for tryptophan in proteins. J Phys Chem B. 2004; 108:4248-4259.

44. Callis PR, Vivian JT. Understanding the variable fluorescence quantum yield of tryptophan in proteins using QM-MM simulations. Quenching by charge transfer to the peptide backbone. Chem Phys Lett. 2003; 369:409-414.

45. Biesso A, Xu J, Muino PL, Callis P, et al. Tryptophan dynamics in GB1 sense very different phenomena on the picosecond and nanosecond timescales. submitted.

46. Xu J, Chen B, Callis P, Rozeboom H, et al. Femtosecond fluorescence dynamics of tryptophan and 5-fluorotryptophan in monellin: slow water relaxation unmasked. Biophys J. 2012; 104(2):681a.

47. Vivian JT, Callis PR. Mechanisms of tryptophan fluorescence shifts in proteins. Biophys J. 2001; 80:2093-2109. [PubMed: 11325713]

48. Elove GA, Chaffotte AF, Roder H, et al. Early steps in cytochrome-c-folding probed by timeresolved circular-dichroism and fluorescence spectroscopy. Biochemistry. 1992; 31:6876-6883. [PubMed: 1322172]

49. Pal SK, Peon J, Bagchi B, et al. Biological water: femtosecond dynamics of macromolecular hydration. J Phys Chem B. 2002; 106:12376-12395.

50. Chen Y, Barkley MD. Toward understanding tryptophan fluorescence in proteins. Biochemistry. 1998; 37:9976-9982. [PubMed: 9665702]

51. Berlett BS, Stadtman ER. Protein oxidation in aging, disease, and oxidative stress. J Biol Chem. 1997; 272:20313-20316. [PubMed: 9252331]

52. Davidson RS. The photodegradation of some naturally occurring polymers. J Photochem Photobiol B. 1996; 33:3-25.

53. Paik DC, Dillon J. The nitrite/alpha crystallin reaction: a possible mechanism in lens matrix damage. Exp Eye Res. 2000; 70:73-80. [PubMed: 10644422]

54. Grossweiner LI. Photochemistry of proteins-a review. Curr Eye Res. 1984; 3:137-144. [PubMed: 6360538]

55. Pirie A. Formation of $N^{\prime}$-Formylkynurenine in proteins from lens and other sources by exposure to sunlight. Biochem J. 1971; 125:203-208. [PubMed: 5168391] 
56. Fukunaga Y, Katsuragi Y, Izumi T, et al. Fluorescence characteristics of Kynurenine and $N^{\prime}$ Formylkynurenine-their use as reporters of the environment of tryptophan-62 in hen egg-white lysozyme. J Biochem. 1982; 92:129-141. [PubMed: 7118867]

57. Grosvenor AJ, Morton JD, Dyer JM. Profiling of residue-level photo-oxidative damage in peptides. Amino Acids. 2010; 39:285-296. [PubMed: 20091070] 


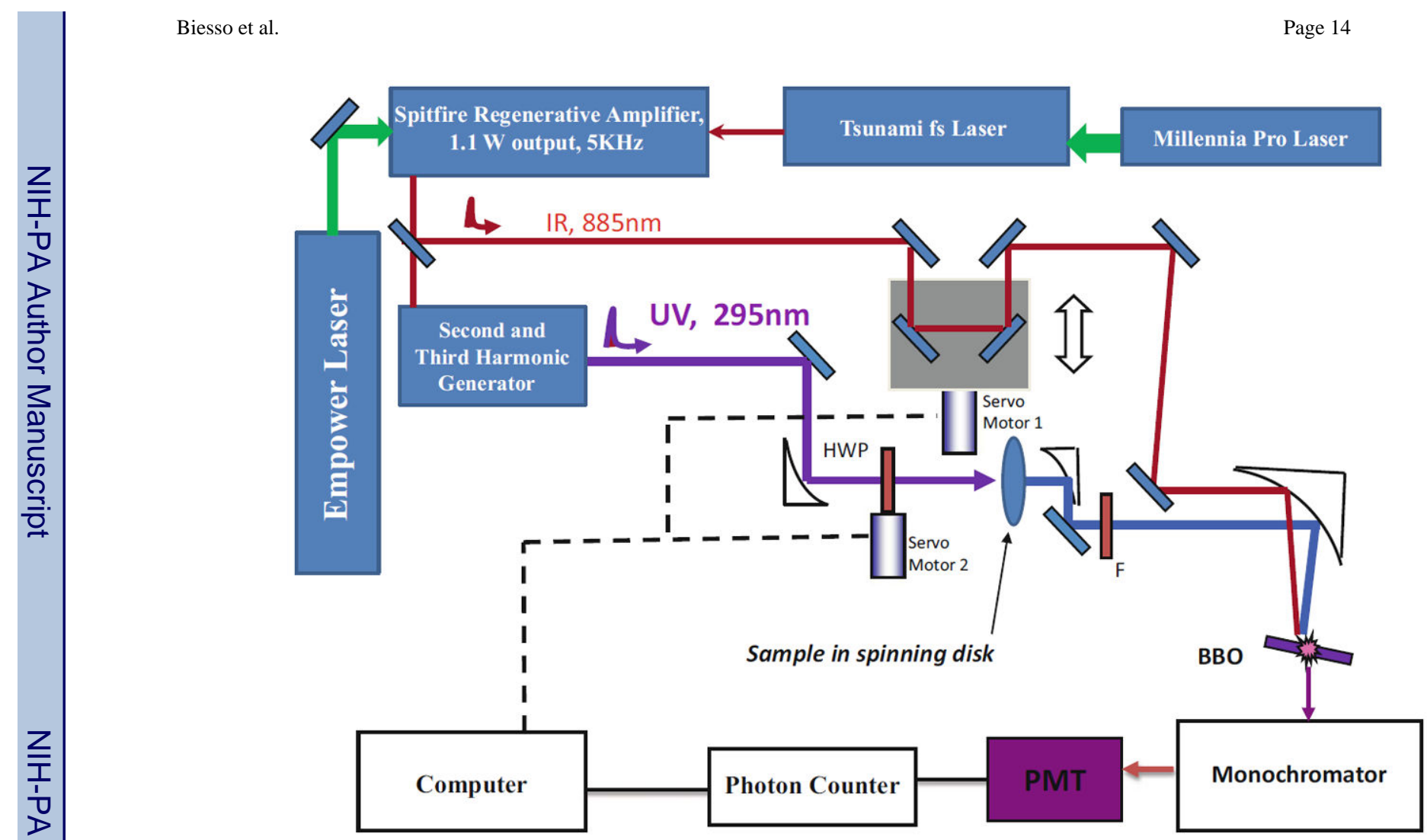

Fig. 1.

A layout of the upconversion spectrometer setup 


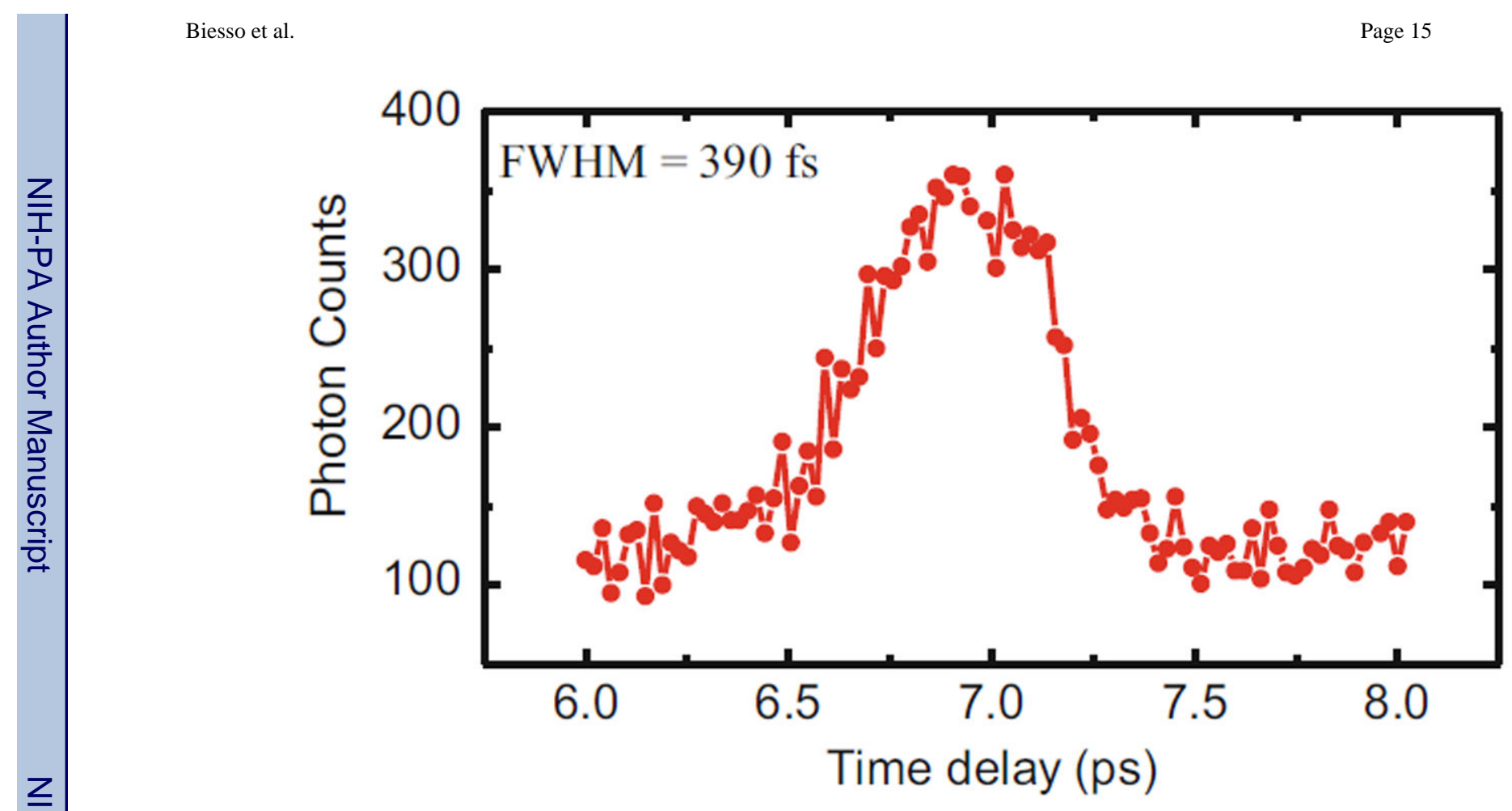

Fig. 2.

A typical snapshot of the response time profile of upconversion spectrophotofluorometer 


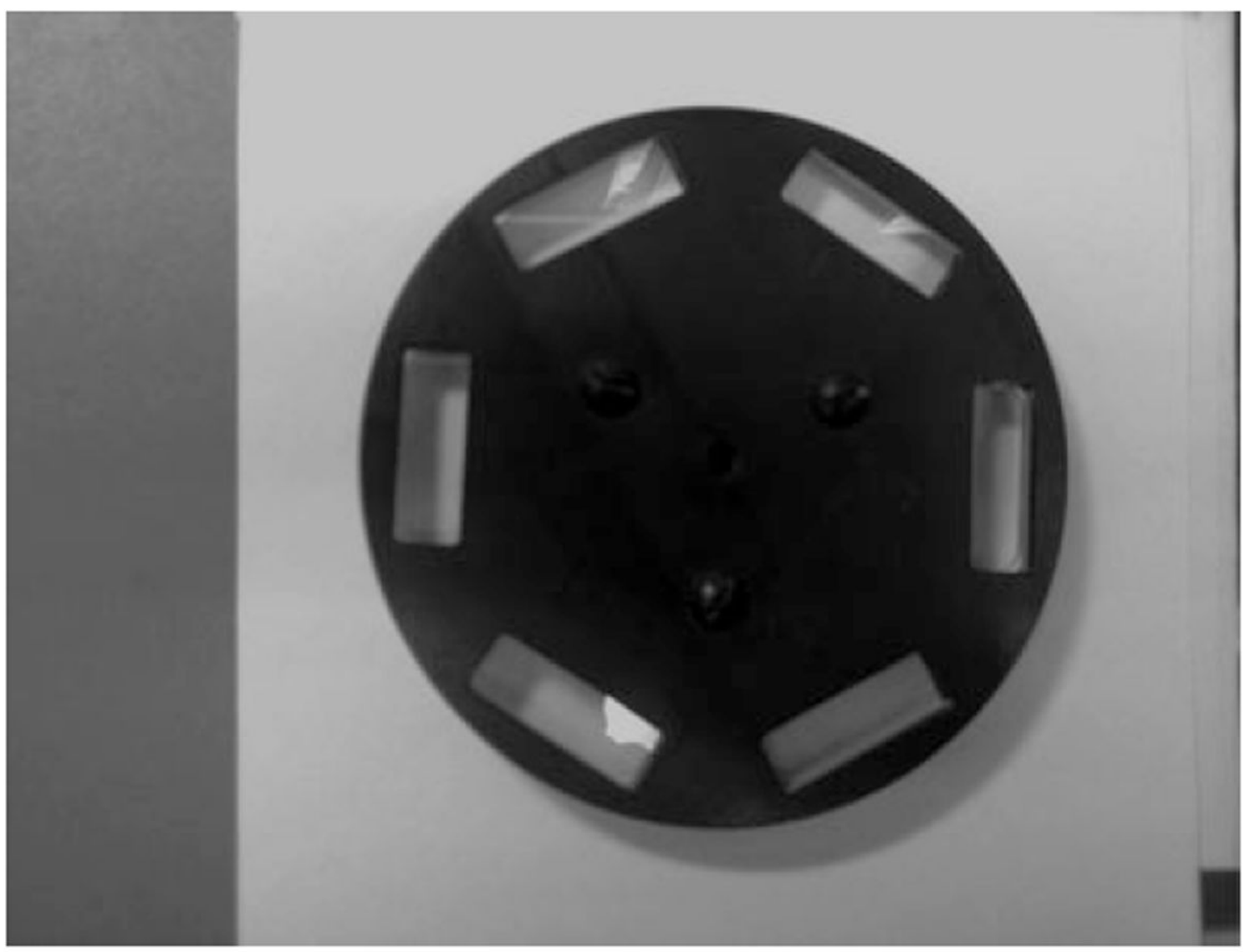

Fig. 3.

Sample holder for fluorescence upconversion measurements. Slotted delrin disk flat cuvette holder for sample spinning. Each slot is about $1 \mathrm{~cm}$ wide $\times 4 \mathrm{~cm}$ long 


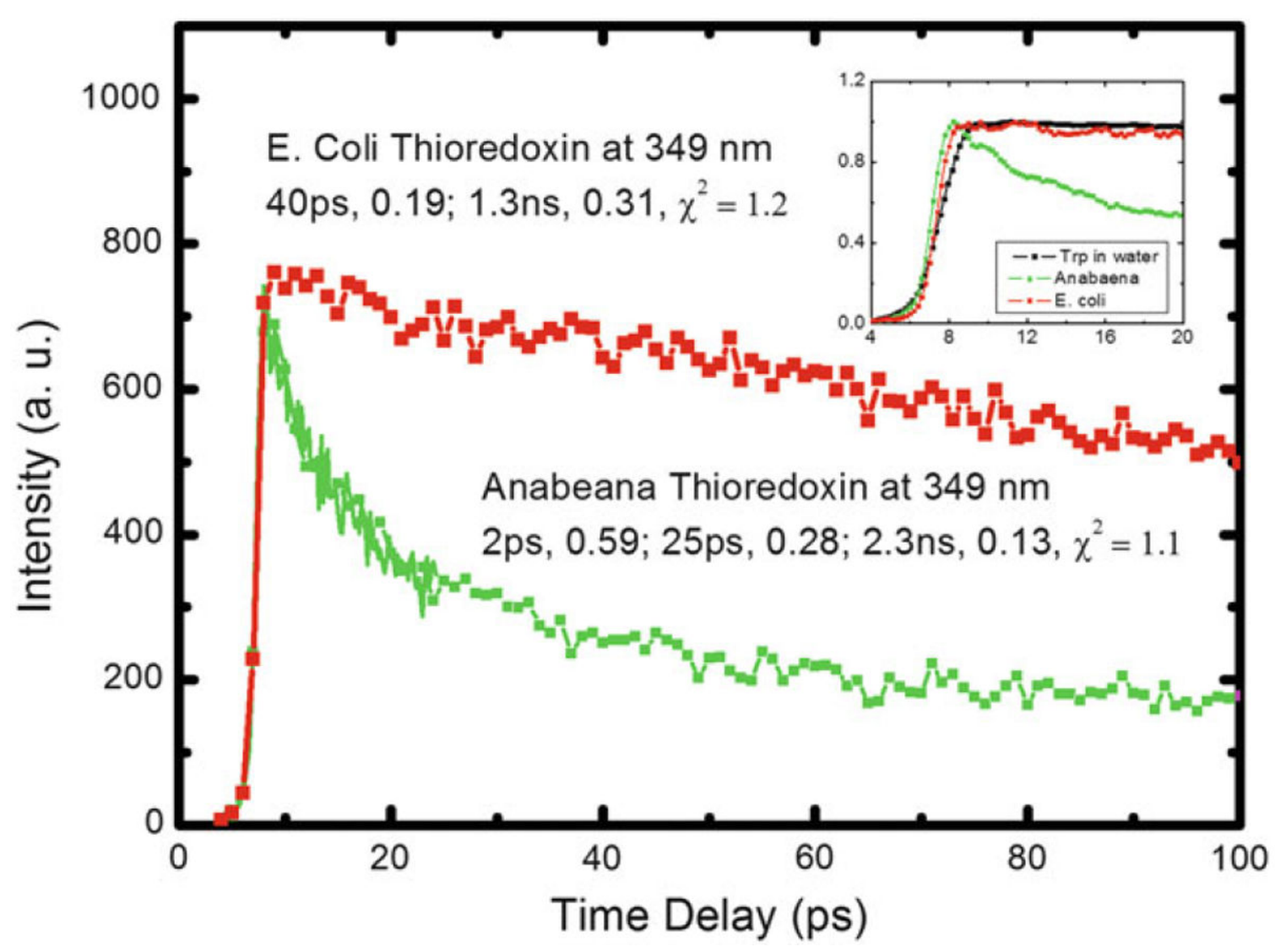

Fig. 4.

Example of a protein decay transient near the emission peak. Thioredoxin proteins in the oxidized state are known to have very low quantum yield, and the mechanism of such quenching has been ascribed to both disulfide bond quenching and to proximate carboxyl groups. Here two different species' thioredoxins are compared at $\lambda_{\mathrm{em}}=349 \mathrm{~nm}$ 
Upconversion signal at $375 \mathrm{~nm}$ for peptide $4 \mathrm{~W}$ after exposure to $295 \mathrm{~nm}$ $+442 \mathrm{~nm}$

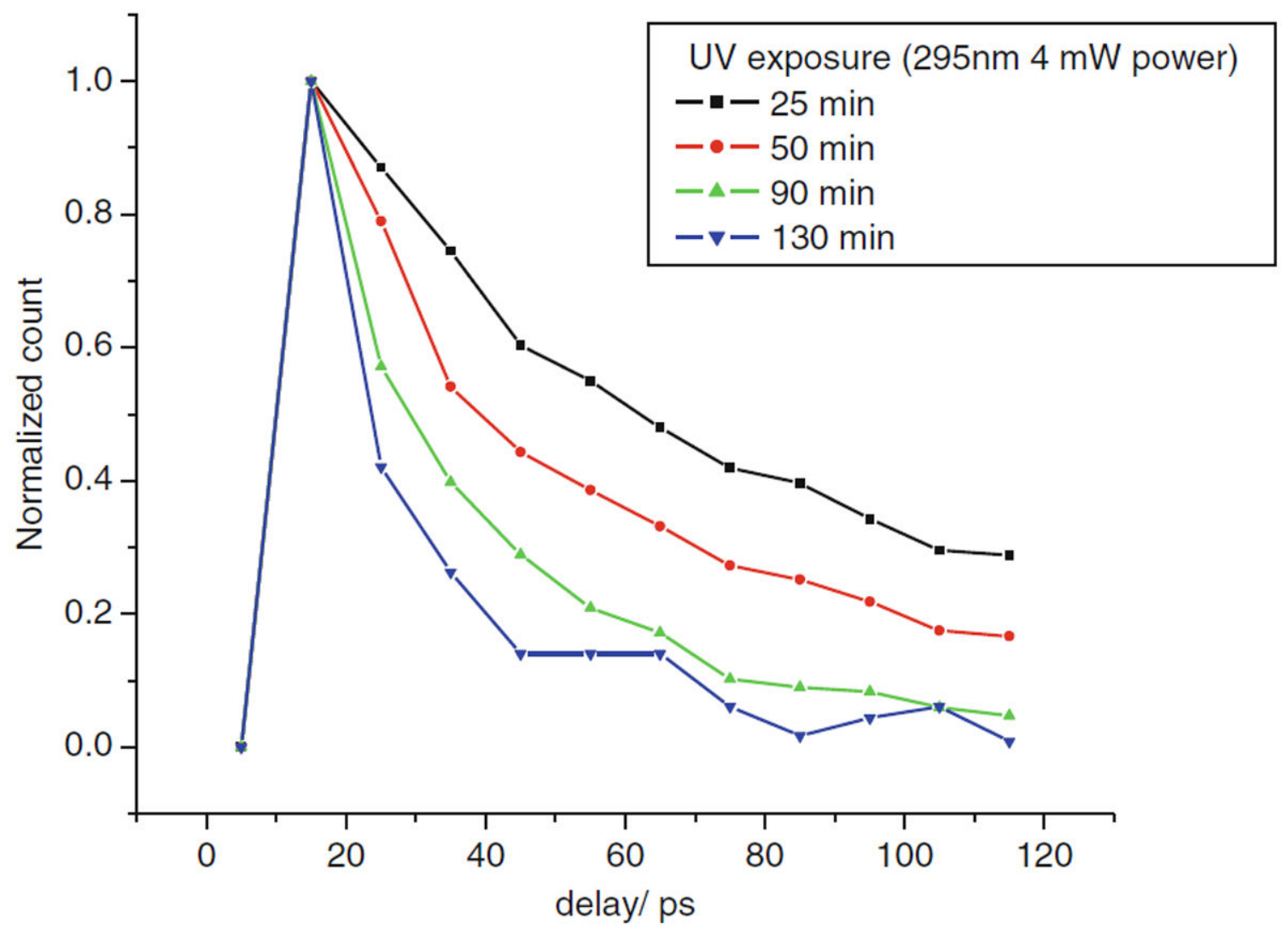

Fig. 5.

Low resolution upconversion traces for a single tryptophan amyloid-related peptide (AcMDVWM) upon exposure to UV pulses. The apparent decay rate at $375 \mathrm{~nm}$ is drastically reduced over time, owing to photodamage 


\section{Pileup losses $5 \mathrm{kHz}$}

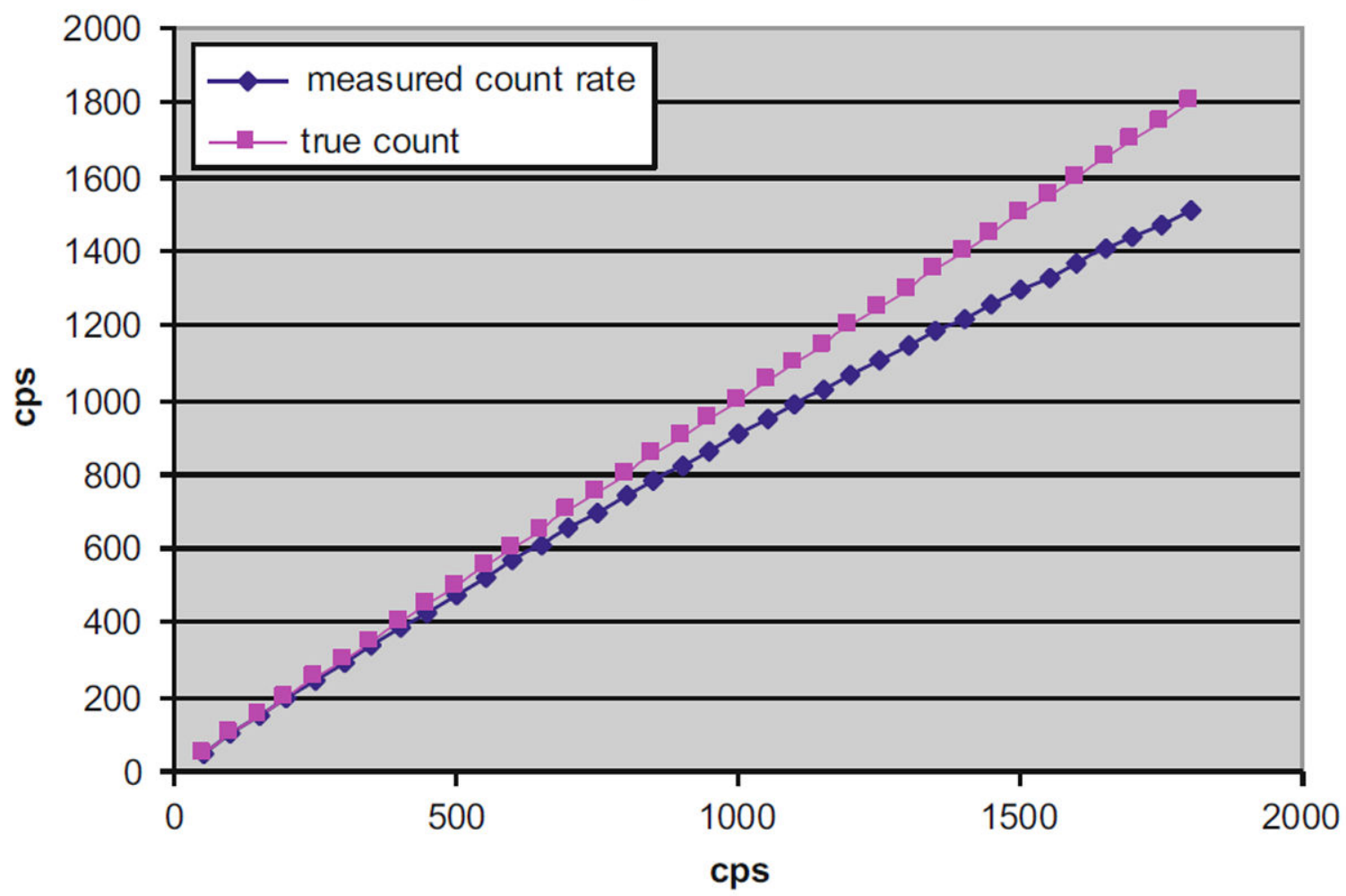

Graph 1.

Poisson statistics of pileup losses for a $5 \mathrm{kHz}$ system as function of the sample photon count. At low photon counts (up to $10 \%$ the rep. rate) the probability of pileup (two photons reaching the detector simultaneously) is low. Such probability increases at higher photon count (30-40\% of the rep rate) especially for a low rep rate system 


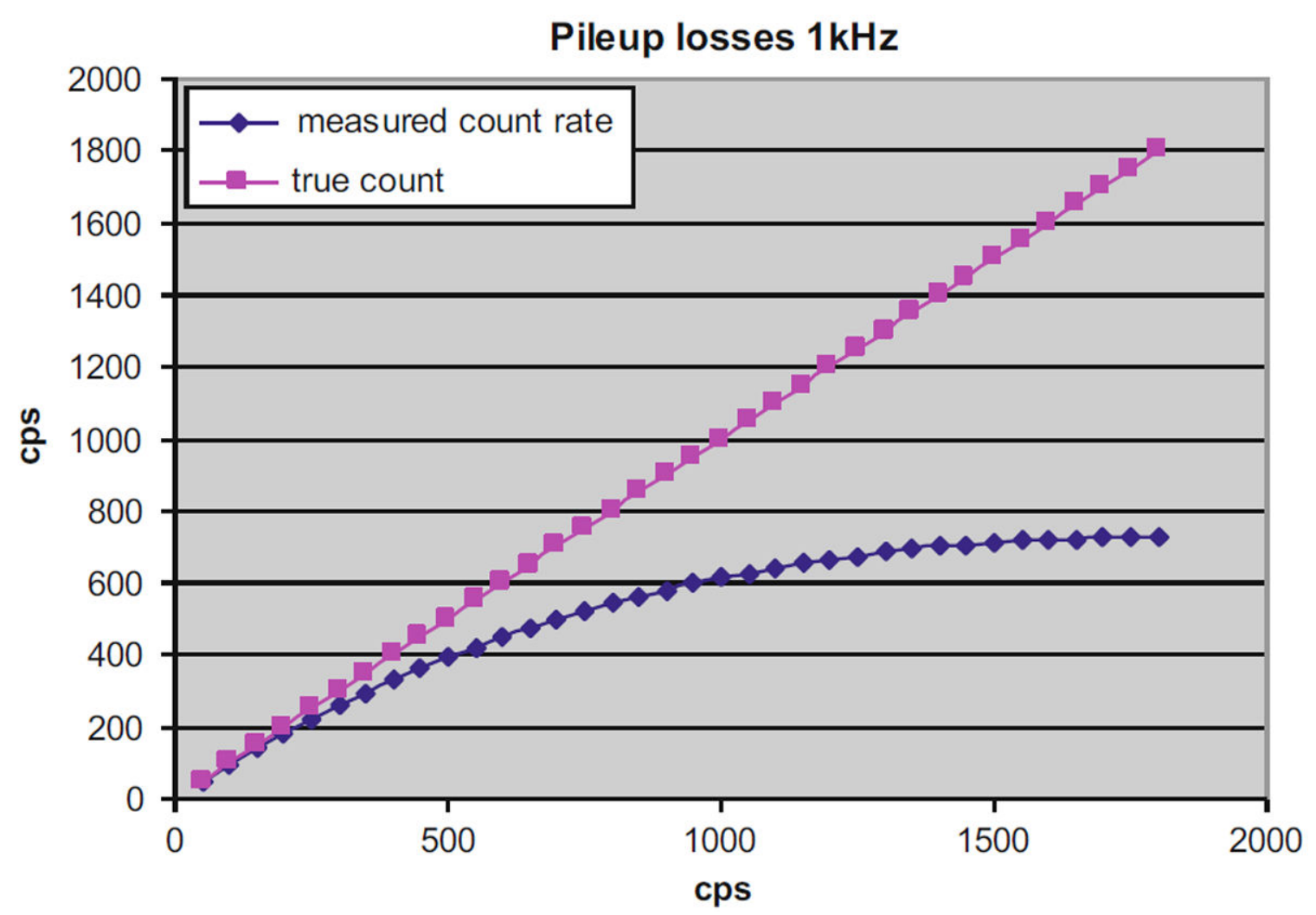

Graph 2.

Poisson statistics of pileup losses for a $1 \mathrm{kHz}$ system as function of the sample photon count. At low photon counts (up to $10 \%$ the rep. rate) the probability of pileup (two photons reaching the detector simultaneously) is low. Such probability increases at higher photon count (30-40\% of the rep rate) especially for a low rep rate system 